\title{
Estrategias y motivaciones en las experiencias educativas de estudiantes de la Universidad Pedagógica Veracruzana: un análisis durante el confinamiento social
} \author{
analysis during social confinement \\ Érick Hernández Ferrer \\ Universidad Pedagógica Veracruzana - México \\ Veracruz, México \\ erick.hernandezfe8o@msev.gob.mx \\ Oscar Valencia Aguilar \\ Universidad Pedagógica Veracruzana - México \\ Veracruz, México \\ ovalencia@msev.gob.mx
}

Strategies and motivations in the educational experiences of students of the Universidad Pedagógica Veracruzana: an

\section{RESUMEN}

El presente trabajo tiene como finalidad evidenciar las estrategias y motivaciones que los estudiantes de la Universidad Pedagógica Veracruzana han empleado para continuar sus estudios en el contexto del confinamiento social derivado de la pandemia por el COVID-19, lo cual obligó a los estudiantes a continuar sus estudios por medios electrónicos. De ahí la necesidad de investigar sobre los estudiantes dentro de este contexto pandémico; el qué y cómo están realizando sus estudios es fundamental en términos de prospectiva, puesto que permitirá mejorar sus procesos en un futuro, en tanto que se hasta se ha planteado un escenario de modalidad mixta para la educación superior toda vez que el confinamiento termine. Siendo así, es que se realizó un estudio de tipo exploratorio, empleando una perspectiva multi-metódica, bajo un enfoque etnográfico; para ello se han empleado técnicas cuantitativas y cualitativas, como: encuestas, registros de observación y entrevistas semiestructuradas dirigidas a una muestra de un total de 82 alumnos de la institución señalada de los niveles de licenciatura, maestría y doctorado. En este sentido, se encontraron elementos de importancia para el manejo de actividades escolares en esta ruptura de la normalidad, pues se identificaron como principal estrategia la diversificación de actividades y motivaciones fundamental el apoyo de la familia para continuar los estudios en confinamiento, lo cual nos ha planteado una visión prospectiva en la cual el apoyo institucional se debe orientar en conocer y tomar en cuenta los contextos socio-culturales de sus estudiantes a fin de lograr establecer una modalidad mixta eficiente.

Palabras clave: Enseñanza Superior; Etnología; Pandemia; Política Educacional; Vida Cotidiana

\section{ABSTRACT}

The purpose of this paper is to show the strategies and motivations that students of the Universidad Pedagógica Veracruzana have used to continue their studies in the context of the social confinement derived from the COVID-19 pandemic, which forced students to continue their studies by electronic means. Hence the need to investigate the students within this pandemic context; what and how they are doing their studies is fundamental in terms of prospective since it will allow improving their processes in the future since a mixed modality scenario has even been proposed for higher education once the confinement ends. Thus, an exploratory study was carried out, using a multi-method perspective, under an ethnographic approach; for this purpose, quantitative and qualitative techniques were used, such as surveys, observation records, and semi-structured interviews directed to a sample of a total of 82 students of the above-mentioned institution at the undergraduate, masters and doctorate levels. In this sense, elements of importance were found for the management of school activities in this rupture of normality, since the main strategy identified was the diversification of activities and fundamental motivations, the support of the family to continue studies in confinement, which has given us a prospective vision in which the institutional support should be oriented to know and take into account the socio-cultural contexts of their students in order to establish an efficiently mixed modality.

Keywords: Higher education; Ethnology; Pandemic; Educational Policy; Daily life 


\section{INTRODUCCIÓN}

Conocer quiénes son los estudiantes y cuáles son sus procesos de aprendizaje es un tema fundamental para la investigación educativa y para las propias instituciones escolares para reconocer los efectos que tienen las políticas -entendidas como cursos de acción- en el desarrollo de las prácticas de los estudiantes, esto lo es aún más importante toda vez que nos encontramos en un contexto pandémico derivado de la COVID-19 y que ha implicado un confinamiento social y el cierre total de las escuelas. Así, en este contexto, es que en el marco del Seminario Institucional en la Investigación Social y Educativa (SISE) de la propia Universidad Pedagógica Veracruzana (UPV), nos hemos dado a la tarea de realizar una investigación intitulada: constitución y formación de sujetos universitarios en contextos de crisis; esto con la finalidad de dar a conocer las dinámicas en las que los estudiantes de la UPV se están formado, no solo como estudiantes, sino como sujetos sobredeterminados.

Lo anterior evidentemente en franca relación con la situación actual desatada por la contingencia sanitaria por el COVID-19, la cual evidentemente nos plantea un escenario extraordinario, como desconocido (y más en la educación superior); en este sentido, en el presente trabajo hemos tomado como eje de análisis la pregunta de investigación: ¿cuáles han sido las estrategias y motivaciones de estudio de los estudiantes de la UPV durante la pandemia derivada por la COVID-19?; es por eso que lo aquí presentado se incluye en los estudios sobre los sujetos de la educación, el cual pretende describir la situación en particular.

En este sentido es que, con este texto se integró una tarea de conformar un enfoque teórico-conceptual que permitiera dar cuenta de las distintas acciones de los estudiantes en esta etapa de confinamiento sanitario, para lo cual, en este trabajo se remota la vertiente antropológica, del análisis institucional y de política pública; es importante mencionar que el grupo de trabajo del SISE es inter y multidisciplinario.

Para realizar el análisis se han retomado los planteamientos de la Antropología Crítica de la Acción (ACA) de quien Bensa A. $(2015,2016)$ es su máximo exponente, del Análisis Institucional se retoman los planteamientos de Remedí E. (2008) y Fuentes A., S. (2010, 2012) y respecto al Análisis de Política Pública de quien se retoma a Parsons (2017), Lasswell (1992), Aguilar (1992, 2003), Fuentes (2018), Villarreal (2010).

Con lo anterior con la intención de dar cuenta de la acción de los sujetos entendidos como como personas activas que persiguen fines concretos y que experimentan medios distintos para alcanzarlos (Viqueira, 2016), esto dentro de instituciones concretas como lo es la UPV, y la visión prospectiva tiene como finalidad mejorar los procesos internos a favor de lograr aprendizajes satisfactorios y significativos para los estudiantes.

También se identifica el concepto de políticas educativas, como lo expresaron Zorrilla y Villa que las menciona de la siguiente manera:

[...] conjunto de orientaciones, lineamientos o criterios de carácter estratégico, es decir, destinados a facilitar el logro de determinadas finalidades en las que pueda sustentarse la relevancia, eficacia, eficiencia, impacto o equidad de las decisiones que se adopten y las acciones que se emprendan con el propósito de atender o cambiar los insumos, procesos y productos de un sistema educativo. (2003, p. 32).

Ahora bien, para efectos del presente texto, cabe precisar que es un indicio de análisis de las políticas públicas desde la revisión de sus resultados (postdecisión, como la aplicación, interpretación, entre otros), como lo explica Parsons (2017, pp. 53-59), en función de que se revisa los resultados de la aplicación de las acciones emergentes de la Universidad en el contexto del COVID-19. Por tanto, en este análisis muestra una serie de indagaciones sobre la situación que imperó. Pues nos preocupaba que las características del estudiantado de la UPV no fueran del todo tomadas en cuenta, esto como una forma de apoyar futuras decisiones al respecto y que incluso muchas de las estrategias emergidas en este contexto pudieran perdurar por eficacia en los procesos de aprendizaje en los estudiantes.

Para dar contexto a la investigación, se revisaron estudios en México como el Mérida y Acuña (2020); o, casos internacionales como Álvarez et al (2020); Expósito y Marsollier (2020) en los que muestran elementos 
de análisis de educación básica y se describen problemas de segregación educativa que reflejan el uso de recursos pedagógicos predominantes y advierten que el uso de diferentes tecnologías no significa que se ha superado las desigualdades de acceso a la educación, coinciden de alguna manera con los hallazgos de los resultados del presente estudio. Cabe significar que no se encontró algo específico sobre educación superior con respecto a la estrategias y motivaciones de los alumnos al realizar las actividades escolares durante el confinamiento social.

Después de hacer una revisión de los documentos oficiales emitidos por la Secretaría de Educación Pública (SEP) que fueron publicados a nivel nacional para atender a la comunidad estudiantil de México, estos solo estaban dirigidos para el nivel básico; se encontró en el internet la estrategia Aprende en Casa que implementó la SEP (2020) fue para los niveles de: inicial, preescolar, primaria y secundaria y a través de la Estrategia de Educación a Distancia, se estableció para que “...niñas, niños y jóvenes continuar con sus aprendizajes escolares durante el periodo de contingencia sanitaria nacional a través de las herramientas que ofrecen Google y YouTube para Educación.” (SEP, 2020, p. 1). La cual se integró por transmitir clases en canales televisivos, publicar los materiales de educación y hacer uso intensivo de las tecnologías educativas (clases grabadas en la aplicación YouTube, aulas virtuales mediante Google Classroom, entre otros). La segunda versión de esta estrategia nacional se traslado con características similares; uno de los cambios o adaptaciones se identifican con el uso de otra plataforma electrónica para apoyar a estudiantes y maestros, por ejemplo: Teams de la compañía Microsoft.

Otro elemento de análisis fue el que desarrolló la Secretaría de Educación de Veracruz (2020) "Veracruz Educando a Distancia", en la que se publicaron guías complementarias y el uso de la estación de radio oficial del Estado de Veracruz (Radio-Televisión de Veracruz). En ambos casos no se encontraron elementos para la educación superior, ni tampoco para las instituciones formadoras de docentes. En esta dependencia estatal está disponible la plataforma de Microsoft con el uso de correos electrónicos que tienen la cuenta del servidor: @alumnos.sev.gob.mx.

En este sentido, tomando en cuenta lo anterior y ajustando los elementos a contextos locales, es que este trabajo tiene como objetivo primordial el dar a conocer las estrategias y motivaciones que los estudiantes de la Universidad Pedagógica Veracruzana accionaron al momento de la ruptura con la vida cotidiana derivado de la pandemia del COVID-19 provocando el confinamiento social a lo que se ha denominado ruptura de la normalidad. Cabe destacar que, como política pública educativa de la UPV, ante esta situación, se implementaron tres documentos al interior de la Universidad:

a) Estrategia Académica para la atención de los estudiantes en el período de contingencia: documento publicado ante la medida de "distanciamiento social" atendiendo a la información emitida por la Secretaría de Educación de Veracruz, en aras de salvaguardar la integridad de la comunidad universitaria con medidas para la atención de los Programas Académicos vigentes;

b) Estrategia Académica para los programas de estudio de Licenciatura y Posgrado ante la contingencia sanitaria COVID-19. Fase Dos: A partir del anuncio de la suspensión de sesiones presenciales, la UPV implementó una estrategia académica, a través de la cual se transitó hacia los entornos virtuales de aprendizaje, por lo que se consideró construir un escenario a mediano plazo que permita atender la contingencia con una proyección al término del cuatrimestre y semestre.

c) Estrategia Académica para los programas de estudio de Licenciatura y Posgrado ante la contingencia sanitaria COVID-19 Fase Dos y Tres: Elementos para atender los programas educativos de la UPV de marzo a junio de 2020.

Además, desde otra perspectiva, sirve como elemento para generar investigación educativa, en términos de Reimers y McGinn, "la utilización de investigación [educativa] no solo ayuda a los tomadores de decisiones a elegir cursos de acción sino a identificar cuáles son sus opciones” (2000, p. 10).

\subsection{Contexto institucional de la investigación}

Esta institución de educación superior (IES) atiende a 3,610 alumnos en 2 licenciaturas, 1 especialidad, 6 diplomados, 4 maestrías y 1 doctorado en 15 Centros Regionales (CR) a lo largo y ancho del estado de 
Veracruz (Gobierno del Estado de Veracruz, 2018). Para la atención de las actividades escolares la UPV cuenta con 15 CR: Pánuco, Tantoyuca, Naranjos, Tuxpan, Papantla, Martínez de la Torre, Xalapa, Coatepec, Veracruz, Córdoba, Orizaba, Ciudad Mendoza, Cosamaloapan, San Andrés Tuxtla y Minatitlán (UPV, 2020). Se instalaron estos centros con la finalidad de "llevar la instrucción y formación a los ámbitos marginados en el norte y sur del estado, con beneficio especialmente para los profesores de educación primaria, quienes podrían acudir a dichos núcleos urbanos para formarse y actualizarse” (Ordoñez, 2018, p. 90).

Cabe destacar que, a partir de la función social de la UPV relativa a la formación inicial docente y de la actualización del magisterio, las clases se imparten los sábados de manera presencial en los CR que se encuentran instalados en escuelas de educación básica que no son utilizadas en esos horarios. Estos centros escolares son facilitados por las autoridades educativas para el correcto funcionamiento de la UPV. Este abanico de oportunidades de profesionalización y actualización de docentes en Veracruz ha permitido atender a más de 25 mil docentes a lo largo de su existencia (Ordoñez, 2018, p. 110), más los que están en proceso en este momento y los que ingresarán en los años posteriores.

A lo anterior, es importante señalar que en la UPV se cuenta con un modelo educativo denominado Horizonte Educativo, el cual, involucra a la mediación pedagógica que invita a "desarrollar el pensamiento divergente [...] esencial en el proceso de aprender en el aprender, lo que coadyuva a transitar de la ausencia de la emergencia de un comportamiento efectivo, permanente." (Velasco et al, 2016, p. 139). Este horizonte está fundamentado en el aprender en el aprender, según Velasco et al, cuya tesis establece que para la formación inicial de los docentes se requiere incorporar elementos como la auto-organización, la autonomía, la creatividad, la comunicación, el lenguaje, la intersubjetividad (trabajo colaborativo), la ética, la interculturalidad, las Tecnologías de la Información y la Comunicación (2016, pp. 91-117).

\section{Nota Metodológica}

Para lograr el acercamiento con el referente empírico (los estudiantes de la UPV) desde el SISE se diseño un estudio de tipo exploratorio empleando una perspectiva multi-métodica, bajo un enfoque etnográfico; en tanto que, el denominado Enfoque Multimétodo (EMM), se caracteriza "por la implementación de dos o más procedimientos de para la indagación sobre un mismo fenómeno u objeto de estudio a través de los diferentes momentos del proceso de investigación" (Ruiz Bolívar, 2008, p. 8), que en este caso se trató de las estrategias y motivaciones de estudio de los estudiantes de la UPV en el contexto de la pandemia.

Por tanto, se consideró a la etnografía como el enfoque central de acercamiento a los actores en tanto que "permite dar cuenta de algunos aspectos de las vidas de unas personas sin perder de vista cómo éstas entienden tales aspectos de su mundo" (Restrepo, 2016, p. 16), pues el interés es mostrar quiénes son y cuáles son sus saberes y prácticas al estar en una crisis de larga data, particularmente por la contingencia sanitaria; todo esto nos resultó importante -en términos del Análisis Institucional y de Política Pública (siguiendo a nuestro enfoque teórico-conceptual que coloca la mirada en el sujeto)- para comprender la acción de los estudiantes y poder plantear un horizonte prospectivo para la propia UPV.

Siendo así, es que la investigación responde a un EMM secuencial interparadigmático, ya que, aunque cada estrategia se utilizó de manera independiente responden a la misma orientación epistémica para su posterior interpretación en los resultados mediante el procedimiento de triangulación (Ruiz B., 2008), en la cual se utilizaron recursos provenientes tanto del paradigma cualitativo como cuantitativo y lo cual permitió, desde la combinación de diferentes estrategias metodológica, recolectar diferentes tipos de datos (Schettini y Cortazzo, 2015); como por ejemplo, se diseño un cuestionario el cual se aplicó en línea mediante Google Forms, se realizaron entrevistas y registros de observación, así como análisis documental de los lineamientos vertidos por la propia UPV, esto con la finalidad de poder dar cuenta de esta primera etapa.

El universo de análisis fueron los estudiantes de la UPV, alumnos de los investigadores que pertenecen al SISE en los niveles de licenciatura, maestría y doctorado. Como muestra se tomaron a 82 estudiantes. De esta manera fue que se logró realizar un trabajo de tipo descriptivo-interpretativo; ya que se conjugan datos sobre los sujetos y sus condiciones de estudio, con la interpretación de dichas condiciones respecto a la política planteada por la institución para hacer frente a las estrategias, motivaciones y prácticas 
académicas ante el aislamiento social derivado por la COVID-19.

Así, este instrumento consistió en una encuesta que se aplicó en línea y se configuró a partir de tres ejes específicos, a saber:

1) Generalidades de los actores, con la se buscó dar cuenta de quiénes eran nuestros estudiantes, dónde se ubicaban y las condiciones con las que pasan la contingencia sanitaria;

2) Dimensión Institucional, se buscó conocer todas aquellas acciones que nos parecían importantes que la UPV hiciera a favor de los estudiantes, como: capacitación docente y de estudiantes, así como la implementación de alguna plataforma;

3) Opinión de los actores, se quiso dar cuenta de las percepciones y acciones que los estudiantes construían respecto de la situación y sus estudios, particularmente aquí nos enfocaremos a sus estrategias y motivaciones accionadas durante el inicio del aislamiento social.

El análisis permitió conceptualizar dos dimensiones, a saber: generalidades de la muestra y opinión de los actores, la cual esta última se ha desagregado en dos: estrategias de estudio y motivaciones de los estudiantes; con esto quisimos dar cuenta de las percepciones y acciones que los estudiantes construían respecto de la situación y sus estudios accionadas durante el inicio del aislamiento social.

Por tanto, las 82 respuestas se analizaron mediante el uso del software Atlas.ti y en la que se construyeron 7 categorías analíticas: autogestión y organización de las actividades, diversificación de actividades, aprendizaje emergente, sobreposición de actividades presenciales, aprendizaje personal, profesión e ingresos y cumplimiento de metas.

\section{Resultados}

\subsection{Descripción de la muestra}

Lo primero que se debe señalar es que, del total de la muestra, 62 fueron mujeres ( $76 \%$ ) y 20 varones (24\%), cuyas edades van de los 18 años a más de 40; de estas, 18 personas se encuentran en el rango de los 18 a los 21 años, 7 de los 22 a los 25 años, 18 de los 26 a los 30 años, 14 de los 31 a los 35 años, 8 de los 36 a los 40 años y 17 con más de los 40 años (Ver Figura 1).

\section{Figura 1. Edad de los aprendientes que integran la muestra}

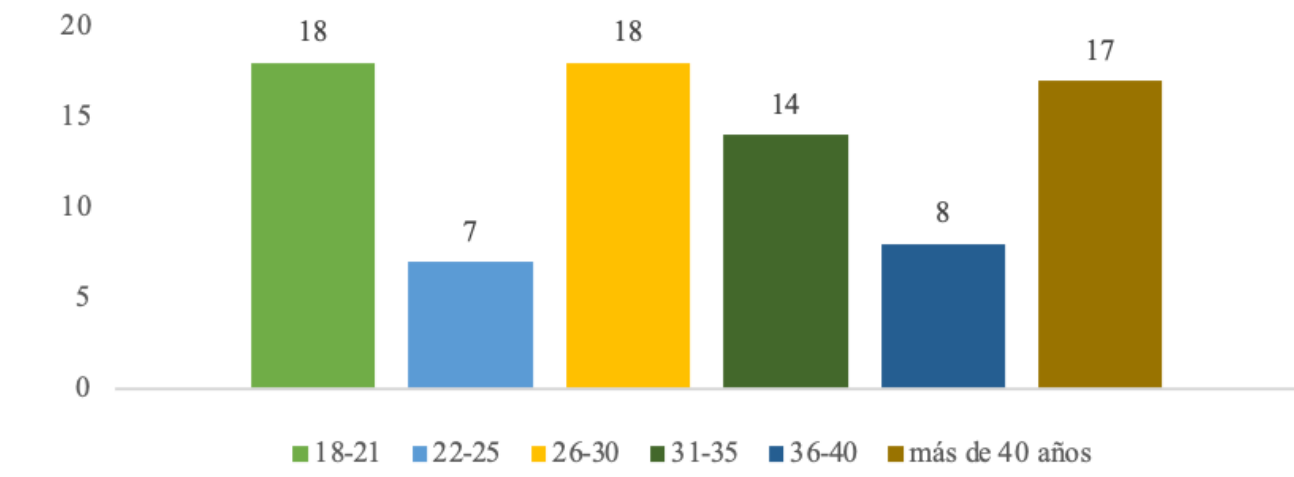

Fuente: Elaborada por los autores para la presente investigación a partir de la Base de datos integrada por el SISE.

En cuanto al CR en el que se encuentran, los datos arrojaron que 56 estudiantes se ubican en Xalapa, 12 en Minatitlán, 8 en Orizaba, 4 en Veracruz y 2 en Córdoba; (Ver Figura 2) los cuales son procedentes de 29 municipios diferentes del Estado de Veracruz y distintas zonas escolares. 
Figura 2. Distribución por Centro Regional los aprendientes que integran la muestra

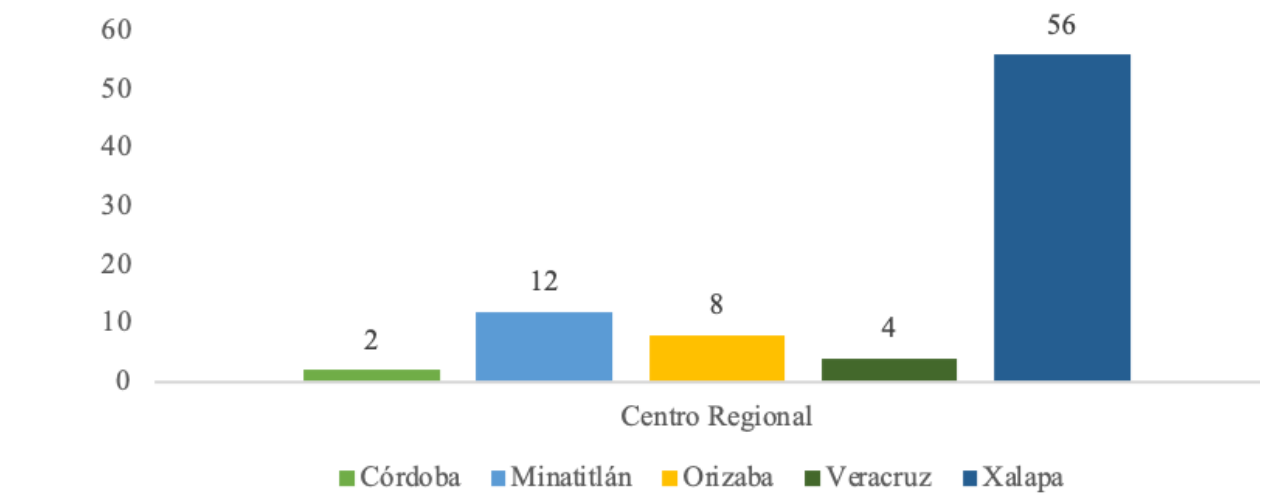

Fuente: Elaborada por los autores para la presente investigación a partir de la Base de datos integrada por el SISE.

En cuanto a su actividad docente, 33 personas (40\%) refirió ser docente frente a grupo, esto en los distintos grados y niveles; estos van desde preescolar (17\%) hasta el nivel superior (6\%), siendo el grado de primaria donde se ubica el mayor porcentaje (46\%). Respecto al confinamiento, de estás 33 personas que son docentes el $63 \%$ continuó realizando sus actividades como docente de forma virtual (Ver Figura 3).

Figura 3. Estudiante de la UPV que es Docente frente a grupo

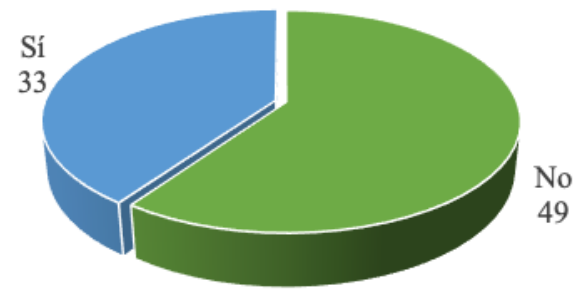

Fuente: Elaborada por los autores para la presente investigación a partir de la Base de datos integrada por el SISE.

Ahora bien, los estudiantes de la UPV que continuaron con actividades como docentes en esta contingencia sanitaria fue $63 \%$ y $37 \%$ de ellos, no están realizando esta actividad en estos momentos (Ver Figura 4).

Figura 4. Estudiante de la UPV que continuó actividades como docente

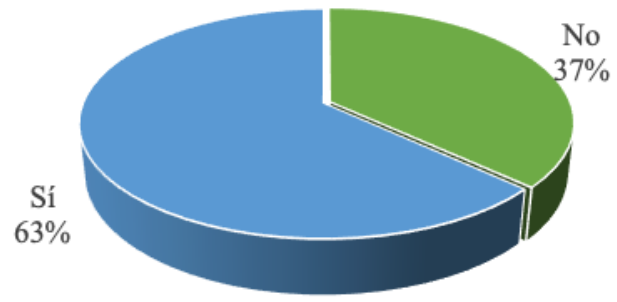

Fuente: Elaborada por los autores para la presente investigación a partir de la Base de datos integrada por el SISE.

De estos 33 estudiantes de la UPV que son docentes frente a grupo, 23 son estudiantes de maestría, 5 de doctorado y 5 de licenciatura (Ver Figura 5). Respecto a su género, 22 son mujeres y 11 varones. 
Figura 5. Distribución de Estudiantes por nivel de estudios en la UPV, que son docentes frente a grupo (con relación al $63 \%$ que continuo con actividades de docencia).

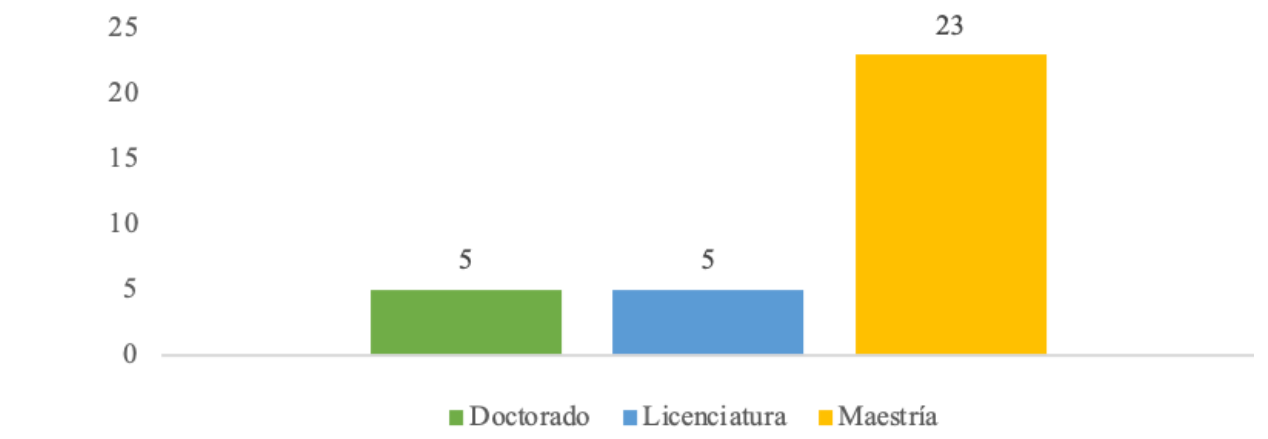

Fuente: Elaborada por los autores para la presente investigación a partir de la Base de datos integrada por el SISE.

Respecto a cómo están pasando la emergencia sanitaria (quédate en casa), el 89\% lo están pasando en residencia habitual y el $11 \%$ en otra residencia; del total de los 82 estudiantes de la muestra, 25 personas la están pasando con tres personas más, 15 con una persona, 14 con cuatro, 12 con dos, 8 con cinco y otras 8 con más de 5 personas (Ver Figura 6).

\section{Figura 6. Con cuántas personas están pasando la emergencia sanitaria.}

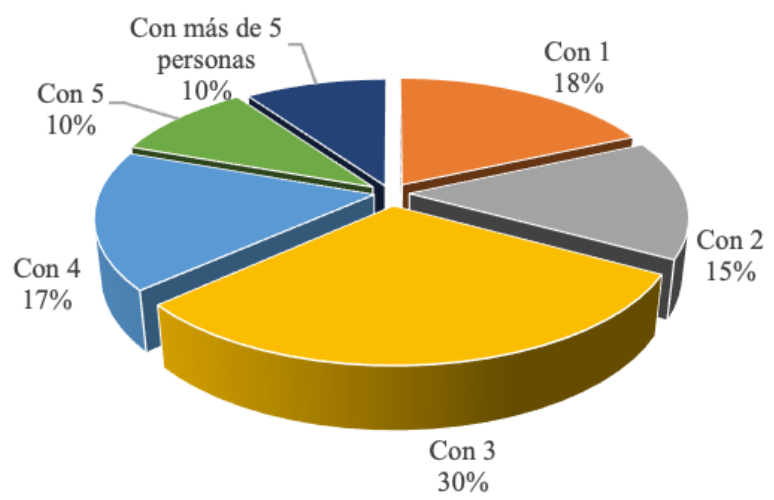

Fuente: Elaborada por los autores para la presente investigación a partir de la Base de datos integrada por el SISE.

\subsection{Estrategias de estudio implementadas por los estudiantes de la UPV durante la pande- mia}

Una de las principales estrategias implementadas por los estudiantes de la UPV se encuentra la autogestión y organización de las actividades, esta consiste en la organización del tiempo de estudio con las de las actividades cotidianas durante el confinamiento; como se pudo ver en el apartado anterior, muchos de ellos comparten equipos y además atienden asuntos familiares, por ejemplo mencionan: "dividir mi tiempo, ya que por las mañanas hasta casi las 4 atiendo a mi grupo de forma virtual y las tareas las realizo por las noches" (Opinión_Estudiante 12, 2020) o bien, “organización de mi tiempo, ya que mi esposo también trabaja desde casa y compartimos la computadora” (Opinión_Estudiante 07, 2020), es por ello que han tenido que autogestionar sus tiempos para la organización de todas sus actividades.

Así, ante este tipo de situaciones han tenido que jerarquizar sus prioridades y programar horarios para el desarrollo de todas las actividades (no solo estudiantiles), señalan: "ajustar mis tiempos y estudiar por las noches" (Opinión_Estudiante 12, 2020), mediante la destinación de una cantidad de tiempo para el desarrollo de las actividades han podido darle un seguimiento académico. 
Otra estrategia de autogestión y organización de las actividades ha sido el mantenerse en contacto con compañeros y maestros mediante el uso de las herramientas tecnológicas (plataformas virtuales, aplicaciones como WhatsApp o Skype) y que al respecto refieren: "he podido realizar todas mis actividades, ya que voy midiendo mis tiempos. Los ratos libres los dedico para entrar a las plataformas" (Opinión_Estudiante 67, 2020) y también “mantenerme en comunicación con mis maestros y compañeros (Opinión_Estudiante 45, 2020).

Punto central de esta estrategia ha sido el denominado aprendizaje autonómo, al respecto mencionaron que elegir un lugar y tiempo adecuados para el trabajo escolar, tener lista las aplicaciones para dialogar con compañeros, muchos por ejemplo realizan "resúmenes, esquemas, mapas mentales, foros de debate en línea con mis compañeros" (Opinión_Estudiante 54, 2020), otros realizan "ejercicios autónomos de búsqueda de información, elaboración de mapas conceptuales” (Opinión_Estudiante 24, 2020).

Otra de las estrategias implementadas ha sido la diversificación de actividades, aquí en tanto que los estudiantes no son solo eso, sino que también son padres o madres, maestros o bien comparten el espacio de confinamiento socia con otras personas, quisimos dar cuenta de sus estrategias. Por ejemplo, refieren: "realizar mis actividades por la noche, para poder hacer mis actividades como mamá durante el día" (Opinión_Estudiante 77, 2020), o bien “organizar mis tiempos entre la casa, mi hijo y la escuela” (Opinión_ Estudiante 33, 2020).

Esto los ha llevado a programar horarios y actividades, pues por ejemplo mencionan: "dedicar unas horas por la noche una vez que concluyen las actividades en la casa, esto para entregar las tareas a tiempo" (Opinión_Estudiante 02, 2020).

La última estrategia es la que hemos denominado como sobreposición de actividades presenciales, esto es, pensar que las actividades son tal cual fueran como en la escuela. Aquí refieren "hacer de cuenta que sigo en clases normales, ya que solo asistía los sábados. Además, sigo solo las instrucciones de las actividades" (Opinión_Estudiante 82, 2020).

Así, muchos mencionan que solo siguen al pie de la letra las instrucciones que los profesores emiten en las plataformas virtuales para el cumplimiento de las actividades estudiantiles, por lo que solo en el horario establecido se dedican al estudio, por ejemplo, refieren: "Ios días sábados los destino a mis clases en línea como si estuviera de forma presencial” (Opinión_Estudiante 42, 2020). Por tanto, una de las principales acciones dentro de esta estrategia es seguir las instrucciones que los docentes establecen para el cumplimiento de las actividades escolares.

Por último, todas las acciones anteriores han permitido lo que denominamos como aprendizaje emergente, ya que muchos de los estudiantes de la UPV tuvieron que aprender a buscar y descargar libros, así como utilizar plataformas electrónicas y distintas aplicaciones. Otros de los aprendizajes emergentes ha sido el investigar por su propia cuenta, por ejemplo, mencionan: "algunas técnicas, como investigar por mi cuenta, leer, escuchar videos, practicar” (Opinión_Estudiante 66, 2020).

\subsection{Motivaciones de los estudiantes de la UPV durante la pandemia}

Las motivaciones de los estudiantes las hemos agrupado en las siguientes categorías: aprendizaje personal, profesión e ingresos, cumplimiento de metas y familia. Dentro de la primera, se hace referencia hacia todas las referencias en las que la motivación están en lograr un aprendizaje que les permita mejorar sus prácticas; así, por ejemplo, hacen referencia a que continúan estudiando para mejorar su práctica docente o bien, para "aprender nuevas formas de educación" (Opinión_Estudiante 17, 2020).

Así, continuar con su formación para logar un aprendizaje refieren que es una forma de superación personal, como lo mencionan: "las ganas de superación" (Opinión_Estudiante 02, 2020). Por tanto, continuar con las actividades académicas por parte de los estudiantes de la UPV es una forma de superación personal, en la que el aprendizaje adquirido es significado como "el deseo de ser docente, de aprender más cosas y de aprender estrategias para un aprendizaje significativo" (Opinión_Estudiante 67, 2020). 
Otras dos motivaciones mencionadas por los estudiantes se refieren a la mejora económica y al cumplimiento de una meta establecida (culminar los estudios) antes del confinamiento; así, mencionan "las ganas de salir adelante y terminar con éxito lo que empecé" (Opinión_Estudiante 73, 2020) o bien "los beneficios que pudiera derivar de ello [culminar los estudios]" (Opinión_Estudiante 23, 2020).

Por último, la motivación más menciona fue con relación a la familia y el apoyo que reciben por el esfuerzo que los estudios requieren, refieren: "me motivan mis hijos, darle un ejemplo que a pesar de las cosas no hay que rendirse. Hay que continuar con el esfuerzo" (Opinión_Estudiante 44, 2020). Así, en este sentido, encontramos que la familia es un punto de apoyo fundamental para los estudiantes de la UPV.

\section{Discusión}

Después de este recorrido exploratorio por las estrategias de estudio y motivaciones de los estudiantes de la UPV para continuar sus estudios durante la pandemia derivada por la COVID-19, es posible advertir que si bien esta situación ha sido complicada para todos los sectores, en particular en la UPV se respondió de manera inmediata y realizó actividades emergentes para trasladar lo presencial a medios virtuales (actividades en línea y vía plataformas electrónicas: Zoom, Google Classroom, Edmodo, entre otros); la estrategia de emergencia la UPV, posiblemente por la premura del tiempo, solo hizo una adaptación de los contenidos que se abordaban de forma presencial a los medios electrónicos mencionados, faltándole un enfoque de enseñanza más adecuado.

Un asunto de suma importancia que este análisis inicial de política pública desde la vertiente posdecisional, lo que implicó la identificación de las acciones que la UPV tomó para dar continuidad a los procesos educativa, fue posible dar cuenta elementos que indican cierta innovación, flexibilidad y empatía para la atención de los alumnos (aunque esto de forma espontanea, es decir, no estaban contempladas en ningún documento institucional); así los docentes utilizaron esas distintas plataformas para tener comunicación continua con los estudiantes.

En relación con lo anterior, sería significativo que la UPV bien podría elegir una sola plataforma, de manera que los estudiantes tengan mayor claridad sobre el uso de ella y no tener que estar cambiándose y adaptándose a distintas; esto en términos de ordenamiento institucional sería de gran ayuda, más para aquellos que no están tan integrados a su uso. Al respecto, la propia Secretaría de Educación de Veracruz (a la cual pertenece la UPV) tiene un convenio vigente con la empresa Microsoft quien cuenta con la plataforma Teams, esto ayudaría a formalizar todas las actividades académicas virtuales.

Además de lo anterior, perdió de vista que muchos de sus estudiantes son más que eso, ya que a la vez son hijos, padres de familia y docentes en otros niveles; por lo que también se deben tomar en cuenta otros aspectos de su vida cotidiana para una continuidad aceptable de sus estudios y éstos sean significativos. En este sentido, encontramos en el caso de las Universidades Tecnológicas en México quienes implementan todo institucionalmente un programa de apoyo a los estudiantes para que sus trayectos formativos sean más exitosos, el cual bien se podría diseñar -y no solo para efectos de la contingencia sanitaria- para acompañar a los estudiantes de la UPV; pues como se ha mostrado en los datos anteriores se requiere de un mayor apoyo institucional de manera que se les brinden las herramientas para mejorar sus estrategias de estudio, como lo es la diversificación de actividades de una manera más sistemática.

Por parte de los estudiantes, se observó que aprendieron a reorganizar sus espacios y realizar una autogestión de su aprendizaje, elementos que la propia UPV con su Horizonte Educativo (modelo educativo institucional) plantea, pero que se han desarrollado por la propia voluntad de los estudiantes de continuar sus estudios y no por una acción institucional deliberada. Así, tener un plan y propósito es lo que ha propiciado a los estudiantes a desarrollarlas; aquí, como en muchos otros casos dentro de la educación superior, el apoyo de la familia es la motivación fundamental que el estudiante encuentra para continuar sus trayectos académicos.

Así, en este sentido, los hallazgos si bien son un reflejo de lo sucedido en el momento de ruptura de la normalidad, nos permite señalar la importancia de la establecer un plan que permita una vinculación institucional que, incluso, vaya más allá de la propia pandemia, apuntando a una modalidad mixta para 
la UPV. De esta manera se fomentará y fortalecerá ese aprendizaje autónomo y los procesos de autoorganización, que los estudiantes ya están implementando.

En el estudio de Alcántara, menciona que "instituciones universitarias han cerrado e intentado adoptar el aprendizaje en línea, muy pocas están bien preparadas para hacer este cambio de manera rápida y abrupta." (2020, P. 76). En este caso, la UPV no ha generado elementos de confusión, por lo contrario, ha utilizado el Horizonte Educativo (2016) para generar elementos de auto-organización, con lo que se muestra un contraste de lo que se encontró en este estudio. Además, uno de los contrastes que se puede identificar es lo que sucede en otras latitudes, ya que existen algunas instituciones de educación superior que no han aceptado transitar a esta modalidad: caso de varias facultades de Buenos Aires quienes pospusieron las clases y reorganizaron el calendario académico, bajo el argumento de la calidad en la educación, solo se alcanza con las clases presenciales. Otros ejemplos con tonos diferenciados en Zimbabwe, Malasia, Túnez, Chile y Reino Unido (Alcántara, 2020, pp. 77-78).

Otro contraste que se encontró al revisar otros estudios es que, si bien es cierto al incorporar el uso de las tecnologías en la educación para utilizar el aprendizaje en línea como opción de mayor posibilidad a fin de continuar con las actividades académicas, el cambio de filosofía en la UPV se ha venido aplicado en los últimos años y como lo expresa Umaña-Mata "uso de virtualidad no es sinónimo de educación a distancia" (2020, p. 48). El presente estudio reveló que las personas que se están formando como docentes al interior de la UPV están convencidas que al generar diversas estrategias y encontrar motivaciones diferenciadas para continuar con sus estudios a través de un cambio cognitivo y toman esta opción como un modelo educativo tan válida como la que se utiliza en modalidad presencial.

Se debe considerar en esta discusión se muestra que existen otros estudios en México que ha llevado a cabo la Comisión Nacional para la Mejora Continua de la Educación, pero su enfoque es sobre educación básica y educación media superior, aún no han realizado estudios sobre la educación superior o están en proceso y los resultados se publicarán más adelante. Cabe señalar que, los estudios están enfocados a saber cómo realizan los estudios los alumnos de educación media superior (nivel educativo previo al que se analizó en este estudio) y los resultados son enfocados en que los alumnos utilizan las siguiente estrategias: Resolver individualmente ejercicios o tareas que dejan mis maestras y maestros; Tomar clases virtuales con mis maestras y maestros; Resolver exámenes en línea; Resolver problemas o tareas en equipo; Hacer actividades de la página Jóvenes en Casa; Ver programas educativos de Aprende en Casa en televisión; Participar en algún concurso de Jóvenes en Casa (Comisión Nacional para la Mejora Continua de la Educación, 2020, pp. 17-18).

Por último, todo esto nos muestra la importancia de colocar la mirada en la acción del sujeto, bajo una conceptualización no esencialista nos ha permitido superar el punto de vista normativo, sino también la falacia racionalista y funcionalista (Majone, 2014) con la que tradicionalmente se han abordado los estudios de las políticas en las cuales se asume la tarea como un asunto meramente pragmático y en que los sujetos parecen estar siempre ausentes (Fuentes Amaya, 2010).

\section{Conclusión en visión prospectiva}

Después de este recorrido por las estrategias institucionales y motivaciones que en los estudiantes de la UPV se accionaron al momento de la ruptura con la vida cotidiana, esto derivado por el confinamiento provocado por el COVID-19, ha sido posible observar que la diversificación de las actividades como estrategia y la familia como motivación han sido fundamentales para continuar sus estudios, y con ello lograr sus objetivos escolares.

Los elementos más importantes que se rescatan es el proceso de auto-organización de los maestros y, principalmente, de los alumnos de la UPV a partir de una estrategia que se ha delimitado en diversos momentos en las actividades académicas desde la Rectoría de la Universidad. Si bien es cierto, se percibe como un elemento de mejora todas estas acciones por los resultados de los instrumentos aplicados, se destaca que no se han detenido las acciones para continuar con el proceso de formación inicial de docentes. 
Por lo que, al analizar los casos de la muestra, se alcanzó a verificar que, los actores (estudiantes) y sus condiciones, en lo general son docentes frente a grupo (para la muestra recolectada), además, que son estudiantes de licenciatura y la mayoría están conviviendo con 3 personas. Las estrategias de estudio que implementaron fue la autogestión, así como la organización de las actividades durante el confinamiento. Se destaca que, los alumnos consideran que las clases a la distancia requieren el mismo tiempo, como si estuvieran de manera presencial. El aprendizaje emergente ha tenido una valoración única, ya que desarrollaron técnicas de aprendizaje y lectura.

Si bien es cierto, existen cosas que mejorar en las diversas estrategias académicas, para dar continuidad a las estrategias de estudio y las motivaciones de los alumnos por seguir estudiando en la UPV, el Horizonte Educativo mostró grandes elementos para generar la auto-organización, entre las políticas públicas educativas y las diversas acciones para continuar con la educación superior, se considera que los estudios subsecuentes podrían elaborarse para tener una visión prospectiva, debe tener elementos básicos: visión holística (estudio de la influencia mutua entre las partes y el todo), creatividad (capacidad de dar origen a cosas nuevas y valiosas con mejor modo de hacerlas), participación y cohesión (un objetivo común, con acuerdo de la problemática, analizando las capacidades y potencialidades así como la decisión de cada actor), pertinencia (la unidad conceptual que hace posible que se insista menos en la coordinación explicita de las acciones), convergencia-divergencia (proceso iterativo, con alto grado de Complejidad) así como la finalidad constructora (contestar ihasta dónde llega?, respondiendo en dos aspectos: conceptual y práctico).

Esto se señala dentro del marco del impacto mundial de la educación a consecuencia de la pandemia del COVID-19 ante el cierre de las escuelas ya que, según el Banco Mundial (2020), hasta el mes de abril de 2020 se cerraron escuelas en 180 países y $85 \%$ de los estudiantes en todo el mundo no estaban asistiendo a la escuela, además de la crisis económica que tiene un pronóstico reservado por que se retraerá 3\% la economía mundial y los costos a largo plazo en el sentido de que los estudiantes que abandonan la escuela generarían inequidad en los aprendizajes, poniendo en riesgo a toda una generación a hacer realidad su verdadero potencial (Banco Mundial, 2020, pp. 5-6).

Con estos últimos elementos implica re-pensar o re-configurar a la UPV a fin de retomar y reforzar el Horizonte Educativo (Velasco et al, 2016) para que pueda mostrar en sus planes y programas de estudio desde una perspectiva o modalidad diferente. Tomando en cuenta las estrategias implementadas por los estudiantes de la UPV durante la pandemia para continuar con los estudios, así como las motivaciones de los estudiantes de la UPV durante la pandemia que se reflejaron en el presente estudio; es decir, plantear estrategias que permitan alcanzar los objetivos académicos, buscando implementar procesos creativos con una finalidad constructora.

Estas estrategias que se deben realizar para atender a los estudiantes se recomiendan que sean diversificadas para Licenciatura, Maestría o Doctorado. Esto ayudaría a los aprendientes a identificar la manera están mostrando las motivaciones y estrategias diferenciadas. Es decir, como institución la UPV deberá reconocer los contextos y situaciones en las que sus estudiantes viven de manera que a la distancia las actividades se encuentren mejor ordenadas y sistematizadas, lo que no significa solo trasponer las actividades curriculares en forma virtual, sino todo un abordaje conceptual diferenciado.

Lo anterior, permitiría generar una prospectiva institucional que pueda atender lo cotidiano entendido como lo normalizado con todos los elementos de política pública educativa en clases presenciales; así como atender los tiempos de crisis, que implican la ruptura de la normalidad y que se puedan atender las clases en la virtualidad en línea o distancia, para propiciar una modalidad mixta. 


\section{REFERENCIAS}

Alcántara S, A. (2020). Educación superior y COVID-19: una perspectiva comparada. En H. Casanova Cardiel (Coord.), Educación y pandemia: una visión académica (pp. 75-82). Universidad Nacional Autónoma de México, Instituto de Investigaciones sobre la Universidad y la Educación.

Álvarez, M., Gardyn, N., lardelevsky, A., y Rebello, G. (2020). Segregación Educativa en Tiempos de Pandemia: Balance de las Acciones Iniciales durante el Aislamiento Social por el Covid-19 en Argentina. Revista Internacional de Educación para la Justicia Social, 9(3), 25- 43. https://doi.org/10.15366/riejs2020.9.3.002

Aguilar, L. F. (1992). El estudio de las políticas públicas. Miguel Ángel Porrúa.

Aguilar, L. F. (2003). Problemas públicos y agenda de gobierno. Miguel Ángel Porrúa.

Banco Mundial (2020). Covid-19: Impacto en la educación y respuestas de política pública. Grupo Banco Mundial. Obtenido de: https://g2g.to/41TM

Bensa, A. (2015). Después de Lévi-Strauss. Por una antropología de escala humana. Fondo de Cultura Económica.

Bensa, A. (2016). El fin del exotismo. Ensayos de antropología crítica. COLMICH/CONACULTA.

Comisión Nacional para la Mejora Continua de la Educación (2020). Experiencias de las comunidades educativas durante la contingencia sanitaria por covid-19. Educación media superior. MEJOREDU. https://www.mejoredu.gob.mx/ images/publicaciones/resumen-ejecutivo-ems.pdf

Expósito, C. D., Y Marsollier, R. G. (2020). Virtualidad y educación en tiempos de COVID-19. Un estudio empírico en Argentina. Educación y Humanismo, 22(39). 1-22. https://doi.org/10.17081/eduhum.22.39.4214

Fuentes A., S. (2010). Estudio introductorio. Hacia una analítica de la política educativa. En S. Fuentes A y Cruz P., O. P., Identidades y políticas educativas (pp. 13-38). UPN.

Fuentes A., S. (2012). La construcción del objeto de estudio. Entre la demanda institucional y el oficio de investigar. En Jiménez, M. A. Investigación Educativa. Huellas metodológicas (pp. 219-238). Juan Pablos Editor.

Fuentes, N. F. (2018). Lo político y lo público en las políticas públicas. Una perspectiva político-discursiva. En Fuentes, N. F. Políticas públicas y politicidad en educación. Configuraciones teóricas e investigativas (pp. 23-57). Universidad Pedagógica Veracruzana.

Majone, G. (2014). Evidencia, argumentación y persuación en la formulación de políticas. Fondo de Cultura Económica.

Mérida M, Y., \& Acuña G., L. A. (2020). Covid-19, Pobreza y Educación en Chiapas: Análisis a los Programas Educativos Emergentes. Revista Internacional de Educación para la Justicia Social, 9(3), 61-82. https://doi.org/10.15366/ riejs2020.9.3.004

Lasswell, H. (1992). La orientación de las políticas públicas. En Aguilar, L. V. El estudio de las políticas públicas (pp. 79-103). Miguel Ángel Porrúa.

Ordoñez, C. L. (2018). Universidad Pedagógica Veracruzana (1979-2017) Un horizonte de 38 años. Universidad Pedagógica Veracruzana.

Parsons, W. (2017). Políticas públicas: una introducción a la teoría y la práctica del análisis de políticas públicas. Facultad Latinoamericana de Ciencias Sociales.

Reimers, F., y McGinn, N. (2000). Diálogo informado: El uso de la investigación para conformar la política educativa. Centro de Estudios Educativos-Asociación de Universidades Confiadas a la Compañía de Jesús en América Latina.

Remedí, E. (2008). Sujetos, culturas, procesos en instituciones universitarias. [Ponencia] IV Congreso Internacional de Análisis Organizacional. Red Mexicana de Investigadores en Estudios Organizacionales.

Restrepo, E. (2016) Etnografía: alcances, técnicas y éticas. Envión Editores

Ruiz B., C. (2008). El enfoque multimétodo en la investigación social y educativa: una mirada desde el paradigma de la complejidad. Teré: revista de filosofía y socio-política de la educación. 8(18) 13-28. https://dialnet.unirioja.es/ servlet/articulo?codigo $=2785456$

Schettini, P y Cortazzo, I. (2015). Análisis de los datos en la investigación social: procedimientos y herramientas para la interpretación de información cualitativa. Universidad de La Plata, La Plata. https://doi.org/10.35537/10915/49017

Secretaría de Educación Pública (2020). Estrategia Aprende en Casa. Ciclo 2019-2020. https://aprendeencasa.sep.gob. $\mathrm{mx} /$ site/index

Secretaría de Educación de Veracruz (2020). Veracruz Educando a Distancia. https://www.sev.gob.mx/educando-adistancia/

Umaña-Mata, A. C. (2020). Educación Superior en Tiempos de COVID-19: oportunidades y retos de la educación a distancia. Innovaciones Educativas, 22 (Especial), 36 - 49. https://doi.org/10.22458/ie.v22iEspecial.3199 
Velasco, J. M., Peredo, P., Fuentes, F., González, G., y López, M. (2016). Horizonte educativo. Hacia una pedagogía de la auto-organización. Universidad Pedagógica Veracruzana.

Viqueira, J. P. (2016). Después de Lévi-Strauss. Nexos. https://www.nexos.com.mx/?p=27227

Villarreal, E. C. (2010). Políticas públicas. En Villareal C. E., y Martínez, G. V. H. (Pre)textos para el análisis político. Disciplinas, reglas y procesos. (pp. 257-279) Facultad Latinoamericana de Ciencias Sociales.

Zorrilla F., M y Villa L., L. (2003) Políticas educativas: Educación básica. Educación media superior. Consejo Mexicano de Investigación Educativa.

\section{AUTORES}

Érick Hernández Ferrer. Doctor en Investigación Educativa. Universidad Pedagógica Veracruzana. México. Oscar Valencia Aguilar. Doctor en Política Educativa y Evaluación. Universidad Pedagógica Veracruzana México

\section{Conflicto de intereses}

El autores informan de ningún conflicto de interés posible.

\section{Financiamiento}

No existió asistencia financiera de partes externas al presente artículo.

\section{Agradecimientos}

Agradecimiento a la Universidad Pedagógica Veracruzana por las facilidades otorgadas para recabar información.

\section{Aclaración}

Este artículo es parte de las investigaciones que se desprenden del Seminario Institucional y del Programa de Formación en la Investigación Social y Educativa que está vigente y se conformó en 2020 al interior de la Universidad Pedagógica Veracruzana. 